\title{
EFECTO DE LA SALINIDAD EN LA LIBERACIÓN FOLIAR DE FOSFATO POR LA FANERÓGAMA ACUÁTICA Zostera noltii Hornem. DE LAS MARISMAS DEL RÍO PALMONES (CÁDIZ)
}

\author{
J. L. Pérez-Lloréns' \& F. X. Niel1 ${ }^{2}$ \\ 1. Departamento de Ecología, Facultad de Ciencias del Mar, Universidad de Cádiz, 11510 Puerto Real (Cádiz). \\ 2. Departamento de Ecología, Facultad de Ciencias, Universidad de Málaga, 29071 Málaga.
}

Palabras clave: Fosfato, liberación, salinidad, fanerógamas acuáticas, Zostera noltii.

\begin{abstract}
SALINITY EFFECTS ON PHOSPHATE RELEASE BY LEAVES OF THE AQUATIC ANGIOSPERM Zostera noltii Hornem. FROM PALMONES RIVER ESTUARY (CÁDIZ, SPAIN)
\end{abstract}

We study the et'fects of salinity on the uptake of phospate by roots and rhizomes in the aquatic angiosperm Zostera noltii Hornem., and its possible release through the leaves to the surrounding water. The uptake of phosphate by roots and rhizomes was not affected by different salinity values $(36 \%$ and $18 \%$ ) in the water surrounding the leaves; on the contrary, the foliar release was affeeted. The $6.25 \%$ of the phosphate uptaken belowground organs was released by leaves in the control experiment ( $36 \%$ ) during a 3 hour incubations, against the $57.87 \%$ in the low salinity experiment. Therefore the foliar release of phosphate is low at normal salinities found in the Palmones estuary (34-36\%o), but when salinity values decrease (very rarely in this environment), the foliar release become enhanced 10 times, indicating that under these conditions, individual plants could act as a 'phosphate pump'. Nevertheless the amount of phosphate released to the estuary is low due to the low eoverage of this plants.

\section{INTRODUCCIÓN}

Los ecosistemas dominados por fanerógamas acuáticas, se encuentran entre los más productivos. Existen importantes, aunque complejas, interacciones entre la producción y el suministro de nutrientes necesarios para el crecimiento, ya que una elevada producción precisará una gran demanda nutricional (McROY \& McMILLAN, 1977).

La incorporación de fosfato por las angiospermas acuáticas piicde acontecer tanto a través de las hojas como de las raíces y los rizomas (McROY \& RARSDATE, 1970; RRISTOW \& WHITECOMRE, 1971: DENNY, 1972; PATRIQUIN, 1972; DeMARTE \& HARTMAN, 1974; TWILLEY et al., 1977; BEST \& MANTAI, 1978; PENHALE \& THAYER, 1980; BRIX \& LYNGBY, 1985; PÉREZ-LLORÉNS \& NIELL, 1989; PÉREZ-LLORÉNS et al., 1993). En condiciones normales, la concentración de PRS (fósforo reactivo soluble) en el sedimento supera en gran medida a la del agua superficial, con lo que las partes subterráneas de las plantas actuarán como principales órganos involiicrados eri la toma de este nutriente. Una vez incorporado, será transportado acrópetamente hacia las hojas pudiendo ser liberado al agua por excreción directa ("bomba de fosfato") (McROY et al., 1972) o por descomposición de los tejidos senescerites (McROY et al., 1972, NICHOLS \& KEENEY, 1973; HARRISON \& MANN, 1975; KISTRITZ, 1978: HILL, 1979; PELLIKAAN \& NIENHUIS, 1988; PÉREZ, 1989).

Los trabajos mencionados han sido realizados en comunidades de aguas epicontinentales o marinas, sin embargo prácticamente ninguno ha abordado el estudio de la influencia de las fanerógamas acuáticas en el ciclo del fósforo en sistemas estuáricos. En estos sistemas una variable dc control importante es la salinidad. La salinidad de la masa de agua del estuario del río Palmones es bastante constante (34\%-36\%o), sin embargo existen cambios importantes con distintas frecuencias de mariifestación. Los de mayor frecuencia (diaria) dependen del ciclo mareal y suponen una exposición regular de las plantas a condiciones de emersión-inmersión. Los cambios de menor fre 
cuencia se deben a inundaciones esporádicas por fuertes lluvias y a las avenidas de agua dulce. La capacidad de adaptación de las plantas a la salinidad dependerá de la facilidad de internalizar estos cambios. Los cambios que ocurren más frecuentemente se internalizan más fácilmente que los menos frecuentes, que a menudo son también impredictibles (lluvias intensas). La disminución brusca de salinidad, y de presión osmótica asociada, debe afectar a la fisiología de estas plantas. En el presente trabajo se ha estudiado el efecto de la disminución de la salinidad sobre los procesos de incorporación radicular y rizomática de fosfato y su posterior liberación por hojas "sanas" ("bomba de nutrientes") en la angiosperma acuática Zostera noltii Hornem., a la vez que se ha cuantificado la importancia que

a

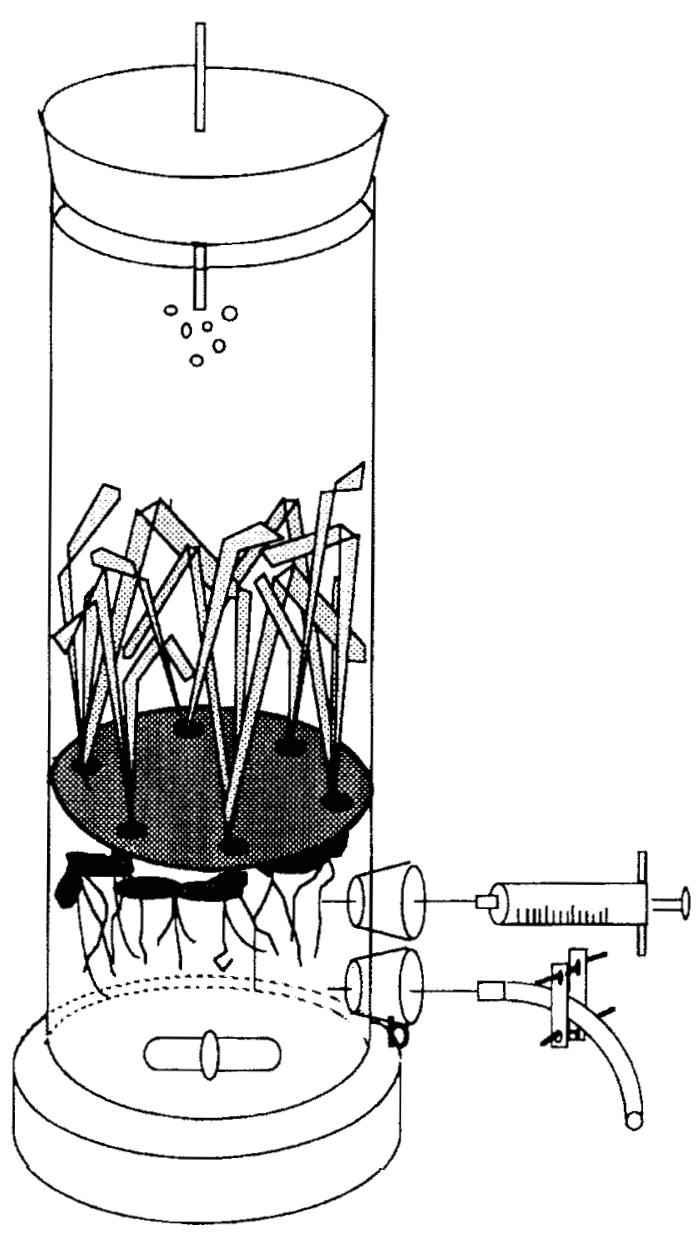

este proceso puede tener en el ciclo del fósforo en el estuario del río Paimones (Cádiz).

\section{MATERIAL Y MÉTODOS}

Las plantas fueron recolectadas en marea baja y se mantuvieron durante tres días en un medio de incubación libre de fosfato (WOELKERLING et al., 1983) en condiciones constantes de temperatura y densidad de flujo fotónico $\left(15^{\circ} \mathrm{C}\right.$ y $150 \mu \mathrm{E} \mathrm{m}^{2}$ $S$ ' respectivamente).

Para la realización de los experimentos se diseñaron unas cámaras de incubación especiales (Fig. 1) con objeto de poder cuantificar la incorporación radicular-rizomática y la liberación

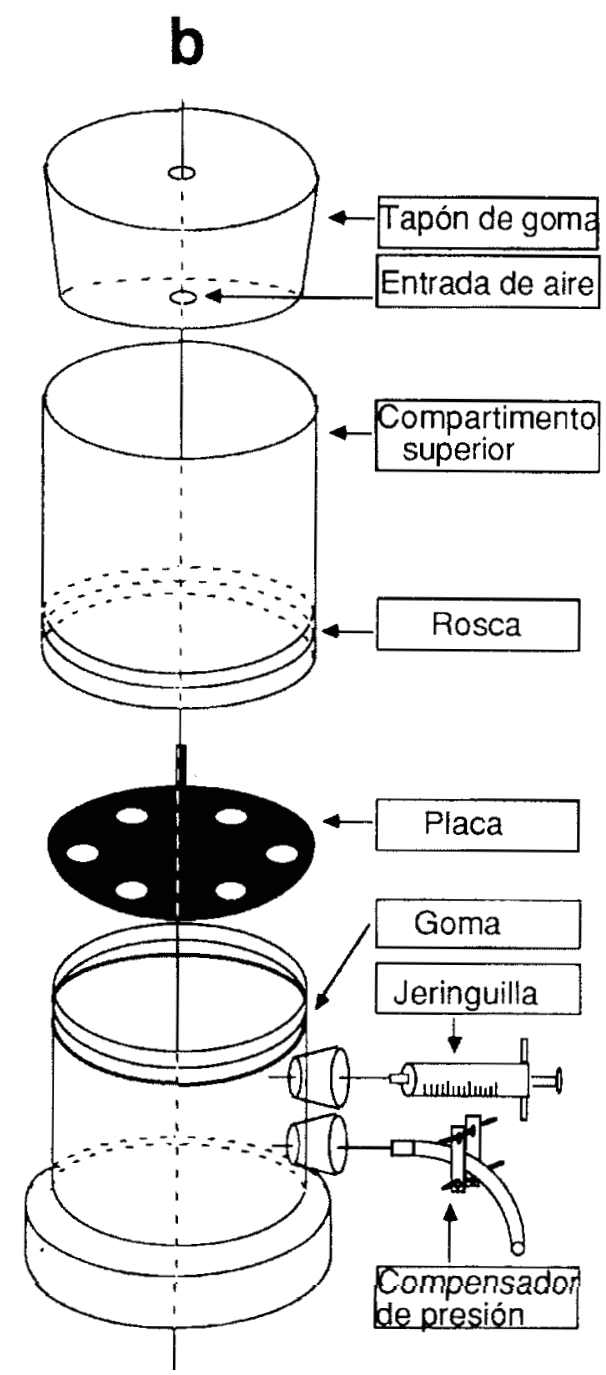

FIGURA 1. a-Cámara utilizada en las incubaciones; b-Detalle de montaje FIGURE 1. a- Experimental incubation chamberi; b- Assembly detail. 
foliar de fosfato. Las cámaras constan de un compartimento superior y otro inferior, separados por un disco de $2 \mathrm{~mm}$ de grosor con 6 orificios por los que se introducen las plantas hasta la altura del peciolo. El compartimento inferior posee dos válvulas, por una se añade o se retiran las muestras de agua y por la otra se compensa la presión ejercida al añadir el pulso de fosfato al inicio de los experimentos.

\section{Montaje de las plantas}

La selección de las plantas era cuidadosa de forma que las hojas y las raices y rizomas estuvieran intactas, montándose seis ejemplares por cámara. Una vez introducidas hasta la altura del pecíolo se procedía a fijarlas utilizando pasta de silicona que servía para obturar completamente los orificios de la placa, único punto de contacto entre el agua contenida en ambos compartimento .

\section{Test de hermet icidad}

Para comprobar que no existían fugas de líquido a través de la silicona, se utilizaron como blanco dos cámaras completamente montadas a las que se añadió, en uno de los compartimentos, un colorante azul (fluoresceina sódica), dejandose en agitación durante cinco días. Al finalizar este período de incubación se pudo constatar que no existían trazas de colorante en el compartimento contíguo. A la vista de este resultado se asume que el único nexo de unión entre los dos compartimentos son las plantas (PERÉZ-LLORÉNS et al., 1993).

Las concentraciones iniciales de fosfato utilizadas fueron de $0,15 \mu \mathrm{M}$ en el compartimento superior y $17 \mu \mathrm{M}$ en el inferior. Esta diferencia de concentración, habitual en el medio natural, obedece al hecho de intentar favorecer el transporte acrópeto de fosfato. Previo al montaje de las plantas, el agua del compartimento inferior era burbujeada durante 15 minutos con nitrógeno con el fin de desplazar el oxígeno y crear las condiciones reductoras que reinan en el sedimento donde estas plantas crecen; también era envuelto con plástico negro para impedir el paso de luz. El agua era agitada permanentemente con un imán para homogeneizar la concentración de fosfato, evitando que se formaran posibles gradientes. En todo momento se utilizó agua con salinidad del $36 \%$. El compartimento superior era burbujeado contínuamente con aire utilizandose dos salinidades distintas: $36 \%$ (control, típica en pleamar) y $18 \%$ (típica en momentos de grandes aportes de agua dulce).

La temperatura e irradiancia fueron $15^{\circ} \mathrm{C}$ y $150 \mu \mathrm{E} \mathrm{m} \mathrm{m}^{-2} \mathrm{~s}^{-1}$. Las muestras de agua para el análisis de la concentración de PRS (FERNÁNDEZ et al., 1985) de ambos compartimentos se tomaron al inicio de los experimentos y a los $5,15,30,60,120$ y 180 minutos. Las tasas de incorporación o liberación de fosfato están normalizadas por el peso seco de las plantas. Las incubaciones se realizaron por duplicado.
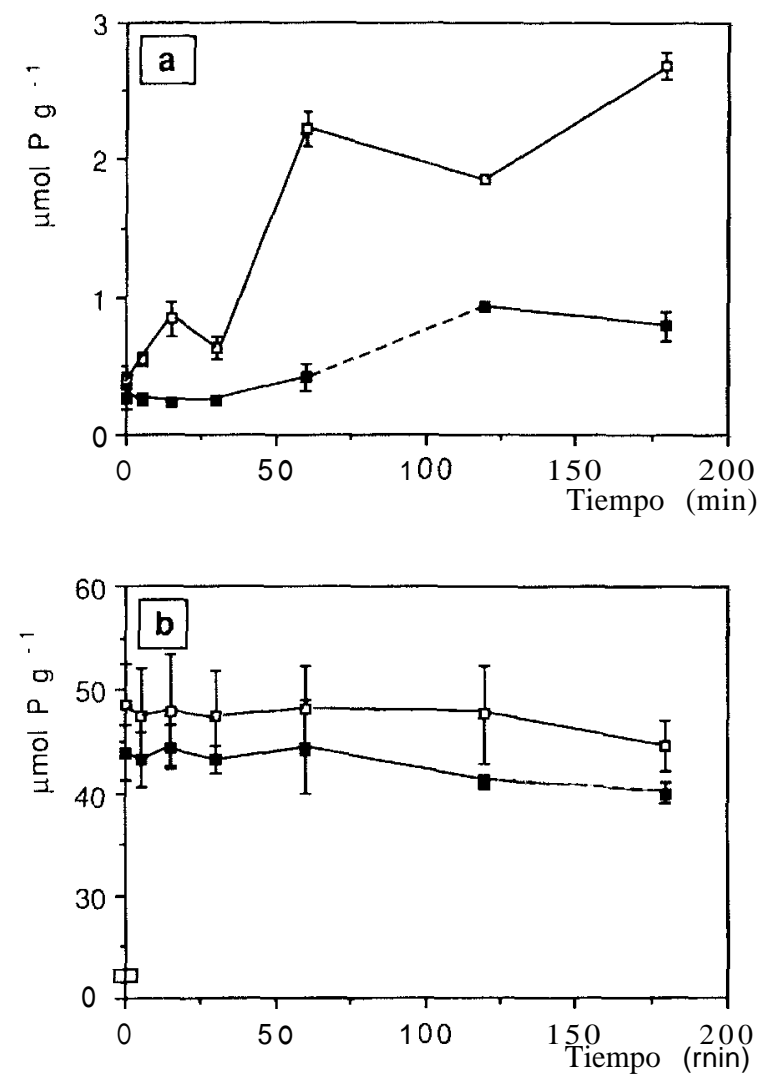

FIGURA 2. Evolución temporal de la concentración de fosfato en el agua del compartimento superior (a) y en la del compartimento inferior (b). En el compartimento foliar se utilizaron dos salinidades distintas: $36 \%$ (controlø) y $18 \%$ ( $\mathbf{\square}$ ). En el compartimento inferior la salinidad se mantuvo siempre en $36 \%$. Los datos son medias de dos réplicas, la desviación standard se representa con barras verticales.

FIGURE 2. Time evolution of phosphate concenuations in the water of the upper compartment (a) and in the lower compartment (b). Two different salinities 36\% (controlø) and $18 \%$ ( $)$ were employed in the upper compartment. Salinity was kept constant $(36 \%$ ) in the lower compartment. Data are averages of two measurements, standard deviations are shown as vertical bars.

\section{RESULTADOS}

En la Fig. 2a se representa la variación en la concentración de fosfato en el agua del compartimento superior, donde se encontraban las hojas de Zostera noltii. En el experimento control (36\% de salinidad) no se registró una liberación foliar de fosfato apreciable hasta una hora después de iniciadas las incu- 
baciones, para ir aumentando gradualmente con pequeñas fluctuaciones. Por el contrario, en el experimento de baja salinidad (18\%o) se detectó, desde el primer momento, un aumento en la concentración de fosfato, incremento muy superior al recogido en el experimento control.

En la Fig. 2b se muestra la variación de la coricentración de fosfato en el agua del compartimento inferior. La salinidad fue siempre de $36 \%$, variando tan solo la del compartimento foliar. Durante la primera hora se observaron, para ambos tratamientos salinos, fluctuaciones en la concentración de fosfato. Para tiempos superiores parece registrarse una incorporación ligeramente mayor en el experimento control (36\%), aunque es dificil de afirmar debido a la escasa replicación del experimento.

En la Fig. 3 se representa, en forma de modelo de compartimentos, el balance neto de fosfato para cada uno de los tratamientos salinos ensayados. Los balances se han calculado para un período de incubación de tres horas. Durante este tiempo, en el experimento control se incorporó a nivel radicular rizomáti-

$36 \%$

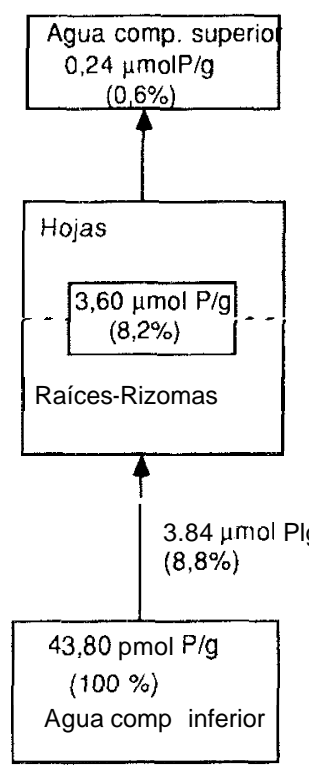

$18 \%$

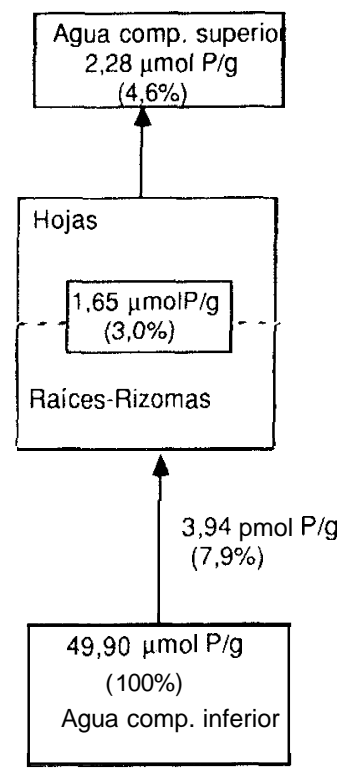

FIGURA 3. Balance neto de fosfato entre los distintos compartimentos considerados para las dos salinidades ensayadas $(\boldsymbol{\Xi 6 \%}$ y y $\mathbf{\square} \%$ ). Los cálculos están basados en incubaciones de 3 horas. Los porcentajes están referidos al fosfato inicial presente en el agua del compartimento inferior. Las cifras que aparecen entre el compartimento foliar y radicular-rizomático quieren significar que, debido al método empleado, no se pudo saber en qué órgano concreto se acumularon.

FIGURE 3. Mass balance of phosphate among the different compartments at the two assayed salinities $(\mathbf{m} \mathbf{3 6 \%}$ y $\mathbf{- 1 8 \%}$ ). Calculations are based on a 3 hour-incubations. Percentages are refered to the initial phosphate in the water of the lower compartment The numbers in foliar and root-rhizonies compartments represent that due to the analytic method employed, it was no possible to know in which part of the plant the phosphate was accumulated. co el $8,8 \%\left(=3,84, \mu \mathrm{mol} \mathrm{P} \mathrm{g}^{-1}\right)$ del fosfato inicial presente en el agua de este compartimento $\left(43,80 \mathrm{pmol} \mathrm{P} \mathrm{g}^{-1}\right)$. El $8,2 \%$ de este $8,8 \%\left(=3,60\right.$ pmol $\left.\mathrm{P} \mathrm{g}^{-1}\right)$ quedó en el interior de las plantas (ya fuera en hojas, raíces o rizomas), y el $0,6 \%\left(=0,24, \mu \mathrm{mol} \mathrm{P} \mathrm{g}^{-1}\right)$ fue liberado, vía foliar, al agua del compartimento superior. En las incubaciones de baja salinidad, el fosfato incorporado por las partes hipógeas fue un 7,9\% $\left(=3,94, \mu \mathrm{mol} \mathrm{P} \mathrm{g}^{-1}\right)$ del fosfato inicial presente, muy similar al del control. De éste $7,9 \%$, el $3 \%\left(=1,65\right.$ pmol $\left.\mathrm{P} \mathrm{g}^{1}\right)$ fue retenido en las plantas y el restante $4,6 \%\left(=2,28\right.$ pmol $\left.\mathrm{P} \mathrm{g}^{-1}\right)$ se liberó vía foliar.

Comparando ambos balances se observa que la incorporación de fosfato a nivel de las raíces y rizomas no se vió afectada por la salinidad existente en el compartimento foliar. Sin embargo, la acumulación de fosfato por las plantas y la liberación vía foliar sí se vieron influenciadas. En las incubaciones de baja salinidad, la acumulación fue una media de 2,7 veces menor que en el control, siendo la liberación foliar casi 10 veces mayor.

\section{DISCUSIÓN}

Los datos que se encuentran en la bibliografía sobre la liberación de fosfato por hojas "sanas" de fanerógamas acuáticas son bastante contradictorios. McROY \& BARSDATE (1970) fueron los pioneros en este tipo de estudios y observaron en Zostera marina una liberación del 33\% del fosfato ( $\left.{ }^{32} \mathrm{P}\right)$ incorporado por las partes subterráneas. En un artículo posterior (McROY et al., 1972) apuntaron que la concentración de fosfato en el agua donde había praderas de esta especie era mayor que en las zonas desprovistas de ellas y estimaron que el fósforo procedente del sedimento y liberado posteriormente por las plantas era $62 \mathrm{mg} \mathrm{P} \mathrm{m}^{2} \mathrm{~d}^{1}$. Estos resultados estimularon el interés en el estudio de las angiospermas acuáticas como pọsibles vectores de transferencia de fósforo desde el sedimento al agua superficial ("bomba de fosfato"). Resultados posteriores obtenidos por otros autores tanto en Zostera marina como en otras especies tanto marinas como dulceacuícolas (Tabla 1) parecen indicar que este efecto de "bomba" no sería, en el mejor de los casos, de tanta magnitud como el observado por los investigadores antes mencionados.

Se han extrapolado las tasas de liberación foliar de fosfato

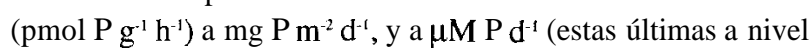
de todo el estuario) utilizando los valores medios de biomasa foliar $\left(\mathrm{g} \mathrm{m}^{-2}\right)$ estimados tanto en invierno (mínimo de biomasa) como en verano (máximo de biomasa) (PÉREZ-LLORÉNS \& NIELL 1993), con intención de compararlas con las obtenidas en Zostera marina (McROY et al., 1972), y tener una idea de su importancia a nivel de todo el estuario (Tabla 1). Somos cons- 
TABLA 1. Cantidades de fosfato liberado, vía foliar, por distintas especies de fanerógamas acuáticas. Los porcentajes están referidos al fosfato incorporado por raíces-rizomas. (clave: *valores calculados a partir de los datos de los autores, $\bullet$ valores correspondientes a verano (primer valor) e invierno (segundo valor), $\mathrm{L}=$ experimentos en luz, $\mathrm{O} \approx$ oscuridad). Para los cálculos de liberación expresados en $\mu \mathrm{M} \mathrm{d}^{-1}$ se ha tomado como volumen del estuario 7,87 x 10 m', y corno cobertura el 20\% (PÉREZ-LLORÉNS, 1991)

TABLE 1. Amounts of phosphate released from healthy leaves of several aquatic phanerogams. Percentages are refered to the phosphate absorbed through the root-rhizome system. (* values calculated from others authors data, $\bullet$ values corresponding to summer (first value) and winter (second), $\mathrm{L}=\mathrm{experiments} \mathrm{carried} \mathrm{out} \mathrm{in} \mathrm{light,} \mathrm{O}=\mathrm{dark}$ ). For calculations of phosphate release expressed as $\mu \mathrm{M} \mathrm{d}^{3}$ it has been assumed that the volume of the estuary is $7,87 \times 10^{6} \mathrm{~m}^{3}$, and the coberture of this species is $20 \%$ (PÉREZLLORÉNS, 1991).

\begin{tabular}{|c|c|c|c|c|c|}
\hline \multirow[t]{2}{*}{ Especie } & \multicolumn{4}{|c|}{ P liberado } & \multirow[t]{2}{*}{ Referencia } \\
\hline & pmol $\mathbf{g}^{-1} \mathbf{h}^{-1}$ & $\%$ & $\mathbf{m g ~ m}^{-2} \mathrm{~d}^{-1}$ & $\mu \mathbf{M ~ d}^{-1}$ & \\
\hline Myriophyllum spicatum & 0 & 0 & 0 & & Bristow \& Whitcombe, 1971 \\
\hline \multirow[t]{2}{*}{ Myriophyllum sp } & - & $0,7-4,6$ & & & Barko \& Smart, 1980 \\
\hline & & 0,010 & & - & Carignan \& Kalf, 1982 \\
\hline Vallisneria spiralis & & 3,6 & - & & Gentner, 1977 \\
\hline Potamogeton sp. & - & $0,3-1,8$ & $-\ldots$ & & Barko \& Smart, 1980 \\
\hline P. pectinatus & 0 & 0 & - & & Welsh \& Denny, 1979 \\
\hline P. Crispus & & 0 & 0 & & Welsh \& Denny, 1979 \\
\hline Najas flexilis & 0,005 & $1,0-2,0$ & - & & Moeller et al, 1988 \\
\hline Ruppiu sp. & $0,04^{*}$ & $4,0^{*}$ & - & & Thursby, 1984 \\
\hline Posidonia australis & $0,005-0,17$ & 一 & - & & Kirkman et al., 1979 \\
\hline \multirow[t]{4}{*}{ Zostera marina } & & 33,0 & - & & McRoy \& Barsdate, 1970 \\
\hline & - & - & 62,0 & & McRoy et al., 1972 \\
\hline & $0,002 "$ & 3,0 & & & Penhale \& Thayer, 1980 \\
\hline & - & 0 & - & & Brix \& Lyngby, 1985 \\
\hline \multirow[t]{5}{*}{ Z. noltii } & 0,050 & - & 7,1 & & Pérez-Lloréns, 1991 \\
\hline & $0,025(\mathrm{~L})$ & $4,0(\mathrm{~L})$ & $3,53-0,75^{\circ}$ & & Pérez-Lloréns, 1991 \\
\hline & & & & $0,012-0,002^{\prime}$ & Pérez-Lloréns, 1991 \\
\hline & 0,080 & 6,2 & $11,3-2,4^{\bullet}$ & 0,05 & Este trabajo $(36 \%$ ) \\
\hline & 0,760 & 57,9 & $107,5-22,8$ & 0,46 & Este trabajo $(36 \%$ ) \\
\hline
\end{tabular}

cientes, sin embargo, de las limitaciones de este tipo de aproximaciones por existir multitud de variables que van a influir tales como la longitud del período experimental, las condiciones de luz/oscuridad, la emersión, la temperatura, la propia manipulación de las plantas al arrancarlas del sedimento, etc. Los valores calculados en el experimento control $(36 \%$ ) están muy por debajo de los obtenidos por McROY et al. (1972) en Zostera marina. La salinidad del estuario del río Palmones es muy constante (34-36\%) aunque pueden producirse, de forma muy esporádica, disminuciones importantes. Por tanto en estas condiciones prácticamente constantes de salinidad, la liberación foliar de fosfato no constituiría un mecanismo importante de aporte de fosfato al agua en este estuario. Además, y considerando las elevadas tasas de incorporación foliar de fosfato que presenta $Z$. noltii (PÉREZ-LLORÉNS, 1991), la baja tasa de descomposición que tienen sus hojas (PÉREZ, 1989), y las elevadas concentraciones $(0,5-15 \mu \mathrm{M})$ de este elemento en la columna de agua (CLAVERO et al., 1991) conllevaría a que estas plantas más que actuar como fuentes de fosfato actuarán más bien como sumideros de este elemento, formando parte del denominado filtro estuárico (SHORT, 1987).
Sin embargo, y a la vista de los resultados, se observa que una disminución brusca de la salinidad, típica de períodos esporádicos de intensas lluvias, incrementa en un orden de magnitud la tasa de liberación foliar de fosfato, reflejando efectos a nivel fisiológico. Existen investigaciones que recogen el efecto negativo de las bajas salinidades sobre la tasa de fotosíntesis de Zostera marina (BIEBEL \& McROY, 1971) y en la merma de poblaciones estuáricas de Halophila ovalis (HILLMAN \& McCOMB, 1988), pudiendo estar ocasionado por una disminución de la concentración interna de nutrientes debido al incremento de su liberación foliar en situaciones de baja salinidad.

Las avenidas de agua dulce además de producir una disminución importante en la salinidad aumentarían la concentración de fósforo en el estuario como consecuencia de la erosión y el lavado de la cuenca y la resuspensión del sedimento del estuario. En estas condiciones la cantidad de fosfato liberado por las plantas sería presumiblemente insignificante en comparación con el fósforo aportado por estas vías. Como ya he referido, un aumento en la concentración de fosfato en la columna de agua supone que las plantas actúen como sumideros netos de este 
nutriente como consecuencia del incremento de las tasas de incorporación foliar de este elemento.

\section{AGRADECIMIENTOS}

Este trabajo ha sido posible gracias a la financiación de la CICYT (proyecto PBI 86-0677). El primer autor quiere mostrar su agradecimiento a Patrick De Visscher y al Profesor Nienhuis del Netherlands Institute of Ecology (Yerseke, Holanda) por las facilidades a la hora de construir las cámaras de incubación.

\section{REFERENCIAS}

BARKO, J. W. \& R. M. SMART, 1980. Mobilization of sediment phosphorus by submersed freshwater macrophytes. Fresh. Biol. 10: 229-238.

BEST, M. D. \& K. MANTAI, 1978. Growth of Myriophyllum: sediment or lake water as the source of nitrogen and phosphorus. Ecology 59: 10751080.

BIEBEL, R. \& C. P. McROY, 1971. Plasmatic resistance and rate of respiration and photosynthesis of Zostera marina at different salinities and temperatures. Mar. Biol. 8: 48-56.

BRISTOW, J. M. \& M. WHITECOMBE, 1971. The role of the roots in the nutrition of aquatic vascular plants. Am. J. Bot. 58: 8-13.

BRIX, H. \& J. E. LYNGBY, 1985. Uptake and translocation of phosphorus in eelgrass (Zostera marina L.). Mar. Biol. 90: 111-116.

CARIGNAN, R. \& J. KALFF, 1982. Phosphorus release by subnierged macrophytes: significance to epiphyton and phytoplankton. Limnol. Oceanogr. 27: 41 9-427.

CLAVERO, V., F. X. NIELL \& J. A. FERNANDEZ, 1991. Effects of Nereis diversicolor O. F. Muller abundance on the dissolved phosphate exchange between sediment and overlying water in Palmones river estuary (Southern Spain). Estuur. Coast. Shelf Sci. 33: 193-202.

DeMARTE, J. A. \& R. T. HARTMAN, 1974. Studies on absorption of ${ }^{32} \mathrm{P},{ }^{59} \mathrm{Fe}$ and $\mathrm{Ca}$ by water milfoil (Myriophyllum exalbescens, Fernald). Ecology 55: 188-1 94.

DENNY, P, 1972. Sites of nutrient absorption in aquatic rnacrophytes. J. Ecol. 60: 819-829.

FERNÁNDEZ, J. A., F. X. NIELL \& J. LUCENA, 1985. A rapid and sensitive automated determination of phosphate in natural waters. Limnol. Oceanogr. 30: 227-230.

GENTNER, S. R, 1977. Uptake and transport of iron and phosphate by Vallisneria spiralis L. Aquat. Bot. 3: 267-272.

HARRISON, P. G. \& K. H. MANN, 1975. Detritus formation from eelgrass (Zostera marina). The relative effects of frag- mentation, leaching and decay. Lirnnol. Oceanogr. 5: 924-934.

HILL, B. H, 1979. Uptake and release of nutrients by aquatic macrophytes. Aquat. Bot. 7: 87-93.

HILLMAN, K. \& A. J. McCOMB, 1988. The primary production of the seagrass Halophila ovalis in the Swan/Canning estuary, Western Australia. 111. The effect of salinity, temperature and light on $\mathrm{H}$. ovalis in culture, and computer simulation of growth. Aquat. Bot. 32: 320-327.

KIRKMAN, H., F. B. GRIFFITHS \& R. R. PARKER, 1979. The release of reactive phosphate by Posidonia australis seagrass community. Aquat. Bot. 6: 329-337.

KISTRITZ, R. U, 1978. Recycling of nutrients in an enclosed aquatic community of decomposing macrophytes (Myriophyllum spicatum) Oikos 30: 561-569.

McROY, C. P. \& C. McMILLAN, 1977. Production ecology and physiology of seagrasses. In: Seagrass Ecosystems: A Scientific Perspective (C. P. McRoy \& C. Helfferich, eds.) 5388. Marcel Dekker. New York.

McROY, C. P. \& R. J. BARSDATE, 1970. Phosphate absorption in eelgrass. Limnol. Oceanugr. 15: 6-13.

McROY, C. P., R. J. BARSDATE \& M. NERBERT, 1972. Phosphorus cycling in an eelgrass (Zostera marina) ecosystem. Limnol. Oceanogr. 17: 58-67.

MOELLER, R. E., J. M. BURKHOLDER \& R. G. WETZEL, 1988. Significance of sedimentary phosphorus to a rooted submersed macrophyte (Najas flexilis (Willd.) Rostk. and Schmidt) and its algal epiphytes. Aquat. Bot. 32: 261-281.

NICHOLS, D. S. \& D. R. KEENEY, 1973. Nitrogen and phosphorus release from decaying water milfoil. Hydrobiologia 42: 509-525.

PATRIQUIN, D, 1972. The origin of nitrogen and phosphorus for growth of the marine angiosperm Thalassia testudinum. Mar. Biol. 15: 35-46.

PELLIKAAN, G. C. \& P. H. NIENHUIS, 1988. Nutrient uptake and release during growth and decomposition of eelgrass Zostera marina L., and its effects on the nutrient dynamics of Lake Grevelingen. Aquat. Bot. 30: 189-214.

PENHALE, P. A. \& G. N. THAYER, 1980. Uptake and transfer of carbon and phosphorus by eelgrass (Zostera marina L.) and its epiphytes. J. Exp. Mar. Biol. Ecol. 42:113-123.

PÉREZ, M, 1989. Fanerógamas marinas en sistemas estuáricos: Producción, factores limitantes y algunos aspectos del ciclo de nutrientes. Tesis Doctoral. Universidad de Barcelona. 244 pp.

PÉREZ-LLORÉNS, J. L. \& F. X. NIELL, 1989. Emergence and submergence effects on the distributional pattern and exchange of phosphorus in the seagrass Zostera noltii Hornem. Scient. Mar. 53: 497-503. 
PÉREZ-LLORÉNS, J. L., 1991. Estimaciones de biomasa y contenido interno de nutrientes, ecofisiologia de incorporación de carbono y fosfuto en Zostera noltii Hornem. Tesis Doctoral. Universidad de Málaga, 168 pp.

PÉREZ-LLORÉNS, J. L., P. DeVISSCHER, P. H. NIENHUIS \& F. X. NIELL. 1993. Light-dependent uptake, translocation and foliar release of phosphorus by the intertidal scagrass Zostera noltii Hornem. J. Exp. Mar. Biol. Ecol. 166:165-174.

PÉREZ-LLORÉNS, J. L. \& F. X. NIELL, 1993. Seasonal dynamics of biomass and nutrient content in the intertidal seagrass Zostera noltii from Palmones river estuary, Spain. Aquat.Bot. 46: 49-66.

SHORT, F. T, 1987. Effects of sediment nutrients on seagrasses: literature review and mesocosm experiment. Aquut. Bot. 27: 41-57.
THURSBY, G. B, 1984. Nutritional requirements of the submerged angiosperm Ruppia maritima in algae-free culture. Mur. Ecol. Progr. Ser. 16:45-50.

TWILLEY, R. R., M. H. Rrindson \& G. J. Davis, 1977. Phosphorus absorption, translocation and secretion in Nuphar luteum. Litnnol. Oceanogr. 22: 1 022-1 032.

WELSH, R. P. H. y P. DENNY, 1979. The translocation of $32 \mathrm{P}$ in two submerged aquatic angiosperin species. New Phytol. 82: 645-656.

WOELKERLING, W. J., K. G. SPENCER \& J. A. WEST, 1983. Studies on sellected corallinaceae (Rodophyta) and other algae in a defined marine culture medium. J. Exp. Mar. Biol. Ecol. 67:61-77. 